\title{
On Quasisymmetric Space
}

\author{
P. Sumati Kumari ${ }^{*}$, Ch. V. Ramana ${ }^{2}$ and Kastriot Zoto $^{3}$ \\ 1Department of Mathematics, K L University, A.P, India; mumy143143143@gmail.com \\ 2Department of Mathematics, Vignan University, A.P, India \\ ${ }^{3}$ Faculty of Natural Sciences, University of Gjirokastra, Albania
}

\begin{abstract}
In this paper we introduce quasisymmetric space and examine several topological characteristics of this space and establish common fixed point theorems for four self mappings in quasisymmetric spaces.
\end{abstract}

Keywords: Kuratowski Axioms, Quasisymmetric Space.

AMS Subject classification: 54A20, 55M20.

\section{Introduction}

It is substantial that the eminent contraction principle is a salient result in fixed point theory which has been used extensively in many special directions. In modern years many authors have discussed numerous notions of metric/ quasi metric/dislocated metric/dislocated quasi metric/ symmetric space in different ways ${ }^{1-5}$.

A metric space is a special kind of topological space. In a metric space, topological properties are characterized by means of sequences. Sequences are not sufficient in topological spaces for such purposes. It is natural to try to find classes intermediate between those of topological spaces and those of metric spaces in whose members sequences play a predominant part in deciding their topological properties. A galaxy of mathematicians consisting of such luminaries as Frechet ${ }^{6}$, Chittenden ${ }^{7}$, Frink ${ }^{8}$, W. A. Wilson ${ }^{9}$, Niemytzki ${ }^{10}$ have made important contributions in this area. In this paper we investigate the topological characteristics of quasisymmetric space and derive some common fixed point theorems.

\section{Notations and Preliminaries on Quasisymmetric Spaces}

Let $X$ be a nonempty set. Any function $d: X \times X \rightarrow R^{+}$, where $\mathrm{R}^{+}$is the set of nonnegative real numbers is called a distance function $d$ on $X$. We consider the following:

$d_{1}$ : Self distances are zero: $d(x, x)=0$

$d_{2}$ : Distance is symmetric: $d(x, y)=d(y, x)$

$d_{3}$ : Positive distance between distinct points: $d(x, y)=$ $d(y, x)=0 \Rightarrow x=y$

$d_{4}$ : Triangle inequality: $d(x, z) \leq d(x, y)+d(y, z)$

$d$ is called,

(i) Metric if $d$ satisfies $d_{1}$ through $d_{4}$.

(ii) Dislocated metric if $d$ satisfies $d_{2}$ through $d_{4}$.

(iii) Dislocated quasi metric if $d$ satisfies $d_{3}$ through $d_{4}$.

(iv) Symmetric if $d$ satisfies $d_{1}$ through $d_{3}$.

The pair $(X, d)$ is called a metric/dislocated metric/dislocated quasi metric /symmetric space if $d$ is a metric/ dislocated metric/dislocated quasi metric/symmetric on $X$.

Metric spaces are well known. Dislocated (quasi) metric spaces are introduced by Pascal Hitzler ${ }^{2}$ and topological aspects of a dislocated metric spaces and dislocated quasi metric spaces are studied by Sarma ${ }^{3}$ and Sumati ${ }^{4}$. Fixed point theorems on symmetric spaces can be found in ${ }^{11,12}$.

Here we introduce the notion of quasisymmetric space, probe in to the topological aspects and derive some fixed point theorems. 


\section{Definition 2.1}

A distance function on a space $X$ is said to be quasisymmetric and the pair $(X, d)$ said to be quasisymmetric space if $d$ satisfies $d_{1}$ and $d_{3}$ :

$$
d(x, y)=0 \Leftrightarrow x=y .
$$

\section{EXAMPLE 2.2}

For any set $X$ and any $d: X \times X \rightarrow R^{+}$э

$$
d(x, x)=0 \forall x \in X \text { and } d(x, y) \neq 0 \text { if } x \neq y \text { in } X,
$$

$d$ is quasisymmetric on $X$.

\section{EXAMPLE 2.3}

$d(x, y)=|x|+y^{2}$ for $x, y \in R$ defines a quasisymmetric on $R$ which is not symmetric.

\section{DEFINITION 2.4}

Let $(X, d)$ be a quasisymmetric, $\left\{x_{\alpha}\right\}_{\alpha \in \Delta}$ a net in $X$ and $x \in X$. We say that $\left\{x_{\alpha}\right\}_{\alpha \in \Delta}$ converges to $x$ in $(X, d)$ if

$$
\begin{aligned}
\lim _{\alpha} d\left(x, x_{\alpha}\right)=\lim _{\alpha} d\left(x_{\alpha}, x\right) & =0 . \text { In this case we write } \\
\lim _{\alpha} x_{\alpha} & =x .
\end{aligned}
$$

\section{DEFINITION 2.5}

If $A \subset X$ and $x \in X$ we say that $x$ is a limit point of $A$ if there exists a net $\left(x_{\alpha}\right)_{\alpha \in \Delta}$ in $A-\{x\} \ni \lim _{\alpha} x_{\alpha}=x$.

The set of all limit points of $A \subseteq X$ is denoted by $D(A)$.

\section{REMARK}

In a quasisymmetric space $(X, d)$ if $x \in X$, the constant net $\left(x_{\alpha}\right)_{\alpha \in \Delta}$ where $x_{\alpha}=x \forall \alpha \in \Delta$ converges to $x$.

\subsection{Convergence Axioms}

Let $\left(x_{n}\right),\left(y_{n}\right),\left(z_{n}\right)$ be sequences in $X$ and $x, y, z \in X$.

$$
\begin{aligned}
& C_{1}: \lim x_{n}=x, \lim d\left(x_{n}, y_{n}\right)=\lim d\left(y_{n}, x_{n}\right)=0 \\
& \quad \Rightarrow \lim y_{n}=x \\
& C_{2}: \lim x_{n}=x, \lim y_{n}=x \Rightarrow \lim d\left(x_{n}, y_{n}\right)=0 \\
& C_{3}: \lim x_{n}=x \Rightarrow \lim d\left(x_{n}, y\right)=d(x, y) \text { and } \\
& \quad \lim d\left(y, x_{n}\right)=d(y, x) .
\end{aligned}
$$

$$
C_{4}: \lim x_{n}=x, \lim x_{n}=y \Rightarrow x=y
$$

It is simple to verify that $C_{1} \Rightarrow C_{4}$ and $C_{2} \Rightarrow C_{4}$

Above mentioned convergence axioms can be found in $^{16}$. The authors of ${ }^{16}$ highlighted some convergence axioms and proved some implications and nonimplications among them. By using these convergence axioms we prove some theorems.

\section{TheOREM 2.6}

If $(X, d)$ is a quasisymmetric space with $C_{1}$ and $A, B \subseteq X$ then

(i) $D(A)=\phi$ if $A=\phi$

(ii) $D(A) \subseteq D(B)$ if $A \subseteq B$

(iii) $D(D(A)) \subseteq D(A)$

(iv) $D(A \cup B)=D(A) \cup D(B)$

\section{Proof}

(i) and (ii) are obvious.

From (ii), we have

$D(A) \cup D(B) \subseteq D(A \cup B)$. To establish the reverse inclusion,

let $x \in D(A \cup B)$ and $\left(x_{\alpha} / \alpha \in \Delta\right)$ and be a net in $A \cup B-\{x\} \ni$

$$
x=\lim _{\alpha}\left(x_{\alpha}\right) .
$$

If $\lambda \in \Delta \ni x_{\alpha} \in A-\{x\}$ for $\alpha \geq \lambda$ subsequently

$$
x \in D(A) \subseteq D(A) \cup D(B) .
$$

If not for each $\alpha \in \Delta$, choose $\beta(\alpha) \in \Delta \ni \cdot \beta(\alpha) \geq \alpha$ and

$x_{\beta(\alpha)} \in B$. Then $\left(x_{\beta(\alpha)} / \alpha \in \Delta\right)$ is a co-final subnet of $\left(x_{\alpha} / \alpha \in \Delta\right)$ in $B-\{x\}$

and $x_{\beta(\alpha)}=x$, so that $x \in D(B)$

This shows $D(A \cup B) \subseteq D(A) \cup D(B)$. Thus (iv) holds.

To show (iii), let $x \in D(D(A)),\left(x_{\alpha} / \alpha \in \Delta\right)$ be a net in $D(A)-\{x\}$

$$
\Rightarrow \lim _{\alpha}\left(x_{\alpha}\right)=x
$$

For every $i \in N$ choose $\alpha_{i} \in \Delta-\left\{\alpha_{1} \ldots \alpha_{i-1}\right\} \ni d\left(x_{\alpha_{i}}, x\right)<\frac{1}{i}$ and $d\left(x, x_{\alpha_{i}}\right)<\frac{1}{i}$ since $x_{\alpha_{i}} \in D(A)-\{x\}$; there exists a net $\left\{x_{\beta_{i}} / \beta_{i} \in \Delta\right\}$ in $A-\left\{x_{\alpha_{i}}, x\right\} \ni \lim x_{\beta_{i}}=\alpha_{i} . \quad$ Choose $\beta_{i} \ni d\left(x_{\beta_{i}}, x_{\alpha_{i}}\right)<\frac{1}{i}$ and $d\left(x_{\alpha_{i}}, x_{\beta_{i}}\right)<\frac{1}{i}$ 
Then $\lim d\left(x_{\alpha_{i}}, x\right)=\lim d\left(x, x_{\alpha_{i}}\right)=0$ and

$$
\lim d\left(x_{\beta_{i}}, x_{\alpha_{i}}\right)=\lim d\left(x_{\alpha_{i}}, x_{\beta_{i}}\right)=0
$$

Hence by $C_{1}, \lim d\left(x, x_{\beta_{i}}\right)=0$.

Since the net $\left\{x_{\beta_{i}} / i \in N\right\}$ in $A-\{x\}$ converges to $x \in X, x \in D(A)$.

Thus $D(D(A)) \subseteq D(A)$.

\section{COROLlory 2.7}

If $\bar{A}=A \cup D(A)$, the operation $A \rightarrow \bar{A}$ on $P(X)$ satisfies Kurotawski's Closure axioms ${ }^{13}$ and hence $\mathfrak{I}=\left\{A / A \subset X\right.$ and $\left.\bar{A}^{C}=A^{C}\right\}$ is a topology on $X$.

\section{TheOREM 2.8}

If $(X, d)$ through $C_{1}, A \subset X$ is open (i.e $\left.A \in \mathfrak{I}\right)$ if for every $x \in A$ there exists $\delta>0 \ni B_{\delta}(x) \subseteq A$.

\section{Proof}

Suppose $A$ is open in $(X, \mathfrak{I})$. Then $A^{c}$ is closed, i.e $\bar{A}^{c}=A^{c}$.

Suppose $x \in A$ and there is no $\delta>0 \ni B_{\delta}(x) \subseteq A$. Then for every positive integer $n \exists x_{n} \in B_{\underline{1}}(x) \cap A^{c}$.

$$
\Rightarrow \lim x_{n}=x .
$$

$\Rightarrow x \in A^{c}$, contrary to the assumption that $x \in A$.

Hence $x \in A \Rightarrow \exists \delta>0$ э $B_{\delta}(x) \subseteq A$.

Conversely suppose $\forall x \in A \exists \delta>0$ (depending on $x$ ) э $B_{\delta}(x) \subseteq A$.

We show that $D\left(A^{c}\right) \subseteq A^{c}$. Otherwise $x \in A \cap D\left(A^{c}\right)$.

Since $x \in A, \exists \delta>0 \ni B_{\delta}(x) \subseteq A$.

Since $x \in D\left(A^{c}\right)$, there exist a net $\left(x_{\alpha} / \alpha \in \Delta\right) \subseteq A^{c} \ni$ $\lim _{\alpha}\left(x_{\alpha}\right)=x$.

In view of the fact that

$\delta>0, \exists \alpha_{\delta} \in \Delta \ni d\left(x_{\alpha}, x\right)<\delta$ and $d\left(x, x_{\alpha}\right)<\delta$ for $\alpha \geq \alpha_{\delta}$

$\Rightarrow x_{\alpha} \in B_{\delta}(x)$ for $\alpha \geq \alpha_{\delta}$ A contradiction.

Therefore $D\left(A^{c}\right) \subseteq A^{c}$.

\section{Proposition 2.9}

If $(X, d)$, through $C_{1}$ and $C_{3}, x \in X$ and $\delta>0$ then $B_{\delta}(x)$ is an open set, i.e $B_{\delta}(x) \in \mathfrak{I}$.

\section{ProOF}

Assume $y \in B_{\delta}(x)$. If there is no $r>0 \ni B_{r}(y) \subseteq B_{\delta}(x)$, then for several positive integer $n$, then there is $y_{n} \in B_{\frac{1}{n}}(y) \cap B_{\delta}^{c}(x)$.

$\Rightarrow d\left(y, y_{n}\right)<\frac{1}{n}, d\left(y_{n}, y\right)<\frac{1}{n}$ however $d\left(x, y_{n}\right) \geq \delta$ or $d\left(y_{n}, x\right) \geq \delta$.

The set $\left\{n / d\left(x, y_{n}\right) \geq \delta\right\}$ or the set $\left\{n / d\left(y_{n}, x\right) \geq \delta\right\}$ must be infinite. And let $\left\{n_{k} / k \geq 1\right\}$ be an infinite sequence $\ni d\left(x, y_{n_{k}}\right) \geq \delta \forall k$.

Since $\lim d\left(y, y_{n_{k}}\right)=0=\lim d\left(y_{n_{k}}, y\right)$, by $C_{3}$

$$
\lim d\left(y_{n_{k}}, x\right)=d(y, x) \text { and }=\lim d\left(x, y_{n_{k}}\right)=d(x, y) .
$$

$\therefore d(x, y) \geq \delta$. A contradiction.

We get a contradiction in a similar way if the second set is infinite.

Hence $\exists \delta>0$ э $B_{\delta}(y) \subseteq B_{\delta}(x)$.

\section{Proposition 2.10}

If $(X, d)$ satisfies $C_{1}, C_{3}$ then the induced topology $\mathfrak{I}$ is Hausdorff and first countable.

\section{Proof}

Since $B_{\delta}(x)$ is open $\forall \delta>0$ and by Theorem 2.8, $\left\{B_{\frac{1}{n}}(x) / n \in N\right\}$ is a countable open base at $x$, hence $\mathfrak{I}$ is first countable.

Suppose $\mathfrak{I}$ is not Hausdorff then there exist $x, y \in X$, $x \neq y$ and for every $n>0, B_{\frac{1}{n}}(x) \cap B_{\frac{1}{n}}(y) \neq 0$.

If $z_{n} \in B_{\frac{1}{n}}(x) \cap B_{\frac{1}{n}}(y), \lim z_{n}=x$

Since $C_{1} \Rightarrow C_{4}, x=y$, a contradiction.

Hence $\mathfrak{I}$ is Hausdorff topological space.

\section{Proposition 2.11}

$x \in X$ is a limit point of $A \subseteq X$ iff for every $r>0$, $A-\{x\} \cap B_{r}(x) \neq 0$

\section{Proof}

If $x \in D(A)$ there exists a net $\left(x_{\alpha} / \alpha \in \Delta\right) \subseteq A-\{x\} \ni$ $\lim _{\alpha}\left(x_{\alpha}\right)=x$. 
If $\in>0 \exists \alpha_{\epsilon} \in \Delta \ni d\left(x_{\alpha}, x\right)<\epsilon$ and $d\left(x, x_{\alpha}\right)<\in$ for $\alpha \geq \alpha_{\in}$

Thus $x_{\alpha} \in A-\{x\} \cap B_{r}(x)$ whenever $\alpha \geq \alpha_{\in}$.

On the contrary if for every $r>0, A-\{x\} \cap B_{r}(x) \neq \phi$, for every positive integer $n$, there is $x_{n} \ni$.

$$
d\left(x_{n}, x\right)<\frac{1}{n} \text { and } d\left(x, x_{n}\right)<\frac{1}{n} \text { and } x_{n} \in A-\{x\} .
$$

Thus $\lim \left(x_{n}\right)=x$ and $\left\{x_{n}\right\} \subseteq A-\{x\}$.

$$
\Rightarrow x \in D(A) \text {. }
$$

\section{Main Results on Quasisymmetric Space}

We establish coincidence point results for four mappings satisfying the property (E.A) on a quasisymmetric space $(X, d)$ through $C_{1}, C_{2}, C_{3}, C_{4}$ under a few contractive conditions. We prove common fixed point theorems for such self mappings via weak compatibility.

\section{DEFINITION $3.1^{14}$}

Let $S, T: X \rightarrow X$. The pair $(S, T)$ gratify property $(E . A)$ if there exists a sequence $\left\{x_{n}\right\}$ in $X$ э $\lim _{n \rightarrow \infty} S x_{n}=\lim _{n \rightarrow \infty} T x_{n}=t \in X$.

\section{DEFINITION 3.2 ${ }^{15}$}

Let $S, T: X \rightarrow X$. The pair $(S, T)$ is said to be weakly compatible if $S T x=T S x$, Whenever $S x=T x$.

\section{THEOREM $3 \cdot 3$}

Let $A, B, S$ and $T$ be self-mappings on $X \ni$

(1) $A X \subset T X$ and $B X \subset S X$

(2) The pair $(B, T)$ gratify (E.A) (resp., $(A, S)$ satisfies the property $(E . A)$

(3) For any $x, y \in X, d(A x, B y) \leq m(x, y)$. $m(x, y)=\max \{d(S x, T y), \min \{d(A x, S x), d(B y, T y)\}$, $\min \{d(A x, T y), d(S x, B y)\}\}$

(4) $S X$ is closed subset of $X$

Then there is $u, w \in X$ э $A u=S u=B w=T w$

\section{Proof}

In view of the fact that the pair $(B, T)$ gratify the property (E.A) there is a sequence $\left\{x_{n}\right\}$ in $X$, and a point $t$ in $X \ni$

$$
\lim _{n \rightarrow \infty} d\left(T x_{n}, t\right)=\lim _{n \rightarrow \infty} d\left(B x_{n}, t\right)=0
$$

From (1), there exists a sequence $\left\{y_{n}\right\}$ in $X, \ni B x_{n}=S y_{n}$ and hence $\lim _{n \rightarrow \infty} d\left(S y_{n}, t\right)=0$

By $C_{2}, \lim _{n \rightarrow \infty} d\left(T x_{n}, B x_{n}\right)=\lim _{n \rightarrow \infty} d\left(S y_{n}, T x_{n}\right)=0$

In view of the fact that $S X$ is a closed subset of $X$, there exists $u \in X$ э $S u=t$

From (3), we have $d\left(A u, B x_{n}\right) \leq \max \left\{d\left(S u, T x_{n}\right), \min \left\{d(A u, S u), d\left(B x_{n}, T x_{n}\right)\right\}\right.$, $\left.\min \left\{d\left(A u, T x_{n}\right), d\left(S u, B x_{n}\right)\right\}\right\}$

Letting $\mathrm{n} \rightarrow \infty$ and applying $C_{3}$, we get $d(A u, S u)=0$.

By applying $C_{3}, d(S u, A u)=\lim _{n \rightarrow \infty}\left(T x_{n}, A u\right)$

By applying $C_{1}, \lim _{n \rightarrow \infty}\left(T x_{n}, A u\right)=0$

$$
\begin{aligned}
& \Rightarrow d(S u, A u)=0 \\
& \Rightarrow S u=A u
\end{aligned}
$$

In view of the fact that $A X \subset T X$ there exists $w \in X \ni$ $A u=T w$.

Our aim to prove that $T w=B w$. From (3) we have, $d(A u, B w) \leq \max \{d(S u, T w), \min \{d(A u, S u), d(B w, T w)\}$, $\min \{d(A u, T w), d(S u, B w)\}\}$

$$
\Rightarrow d(A u, B w)=0
$$

Now $d(B w, A u)=d(B w, t) \lim _{n \rightarrow \infty} d\left(B w, T x_{n}\right)=0$

Since $\quad d(A u, B w)=d(t, B w) \lim _{n \rightarrow \infty} d\left(B x_{n}, B w\right) \quad$ and $\lim _{n \rightarrow \infty} d\left(B x_{n}, T x_{n}\right)=0$.

$$
\Rightarrow \lim _{n \rightarrow \infty} d\left(T x_{n}, B w\right)=0
$$

Hence $A u=B w$

Hence $A u=S u=B w=T w$

\section{THEOREM 3.4}

Let $(X, d)$ be a quasisymmetric space through $C_{1}, C_{2}$ and $C_{3}$ let $A, B, S$ and $T$ be self-mappings of $X$ э

(1) $A X \subset T X$ and $B X \subset S X$

(2) The pair $(B, T)$ gratify the property (E.A) (resp., $(A, S)$ satisfies the property (E.A).

(3) The pairs $(A, S)$ and $(B, T)$ are weakly compatible,

(4) For any $x, y \in X, x \neq y$ $d\left(A x, B y_{n}\right)=\max \{d(S x, T y), \min \{d(A x, S x), d(B y, T y)\}$, $\min \{d(A x, T y), d(S x, B y)\}\}$ 
(5) $S X$ is closed subset of $X$. (resp., TX is a closed subset of $X$ )

Then $A, B, S$ and $T$ have a unique common fixed point in $X$.

\section{ProOF}

From above theorem, there is $u, w \in X$ э $A u=S u=T w$ $=B w$.

From (3), $A S u=S A u, A A u=A S u=S A u=S S u$ and $B T w=T B w=T T w=B B w$. If $A u \neq w$ then from (4), we have

$$
\begin{aligned}
d(A A u, A u)= & d(A A u, B w) \\
< & \max \{d(S A u, T w), \\
& \min \{d(A A u, S A u), d(B w, T w)\}, \\
& \min \{d(A A u, T w), d(S A u, B w)\}\} \\
= & \max \{d(A A u, A u), 0, d(A A u, A u)\} \\
= & d(A A u, A u)
\end{aligned}
$$

A contradiction.

$$
\therefore A u=w .
$$

Likewise if $u \neq B w$, we will get a contradiction.

Thus $A u=w=S u=T w=B w=u$ and is a common fixed point of $A, B, S$ and $T$.

Uniqueness:

Let $z, w$ be any fixed point of $A, B, S$ and $T$. Then

$$
\begin{aligned}
d(z, w) & =d(A z, B w) \\
& \leq k \max \{d(S z, A z), d(T w, B w), d(S z, T w) \\
& =d(z, w)
\end{aligned}
$$

Which implies $d(z, w)=0$ and $z=w$.

\section{Acknowledgement}

The authors are indebted to Professor I.Ramabhadra Sarma for his precious annotations and suggestions.

\section{References}

1. Schroeder V. Quasi metric and metric spaces, conformal geometry and dynamics, Volume 10, Pages 355-360 (December 26, 2006) applications to probabilistic spaces, Nonlinear Anal., 36 (1999), 331-344.
2. Hitzler P. Generalized metrics and topology in logic programming semantics $[\mathrm{PhD}$ Thesis]. School of Mathematics, Applied Mathematics and Statistics, National University Ireland, University College Cork; 2001 Jan.

3. Sarma IR, Kumari PS. On dislocated metric spaces. International Journal of Mathematical Archive. 2012; 3(1):1-6.

4. Kumari PS. On dislocated quasi metrics. Journal of Advanced Studies in Topology. 2012; 3(2):66-74.

5. Shen Y, Lu M. Fixed point theorems in symmetric spaces and applications to probabilistic spaces. Nonlinear Anal. 2010; 72:527-32.

6. Frechet CF. Surquelques points du calcul fonctionnal. Rendiconti del Circolo Mathematico di Palermo. 1906; 22:1-74.

7. Chittenden EW. On the equivalence of ecart and voisinage. Trans Amer Math Soc. 1917; 18:161-6.

8. Frink CAH. Distance functions and the metrication problem. Bull Amer Math Soc. 1937; 43:133-42.

9. Wilson WA. On Semi Metric Spaces. Am J Math. 1931; 53(2):361-73.

10. Niemytzki VW. On the third axiom of metric space. Trans Amer Math Soc. 1927; 29:507-13.

11. Rao JM, Kumari PS, Zoto K. Some Fixed Point Theorems in Symmetric Spaces. Inform Sci Comput. 2013 Oct; 2013(2): Article ID ISC220913, 08 pages.

12. Kumari PS. Fixed and Periodic Point Theory in Certain Spaces. Journal of the Egyptian Mathematical Society. 2013; 21:276-80.

13. Kelly J. General Topology. D. Van Nostrand Company, Inc; 1960.

14. Aamri M, Moutawakil DE. Some new common fixed point theorems under strict contractive conditions. J Math Anal Appl. 2002; 270:181-8.

15. Jungck G. Common fixed points for noncontinuous nonself maps on non-metric spaces, Far East. J Math Sci. 1996; 4:199-225.

16. Sarma IR, Rao JM, Kumari PS, Panthi D. Convergence Axioms on Dislocated Symmetric Spaces, Abstract and Applied Analysis. 2014; 2014:1-7. Article ID 745031. doi:10.1155/2014/7450317 